\title{
Comparison of the effectiveness of a
} tailored cognitive behavioural therapy with a supportive listening intervention for depression in those newly diagnosed with multiple sclerosis (the ACTION-MS trial): protocol of an assessor-blinded, active comparator, randomised controlled trial

Litza Kiropoulos ${ }^{1,2^{*}}$ (D) Trevor Kilpatrick ${ }^{2,3}$, Tomas Kalincek ${ }^{2}$, Leonid Cherulov ${ }^{3}$, Elizabeth McDonald ${ }^{4}$, Tissa Wijeratne ${ }^{5}$, Jennifer Threader ${ }^{1}$, Vanja Rozenblat ${ }^{1}$, Neil Simpson-O'Brien ${ }^{6}$, Anneke Van Der Walt ${ }^{7}$ and Lisa Taylor ${ }^{2}$

\begin{abstract}
Background: Multiple sclerosis (MS) is an unpredictable, chronic neurological disease accompanied with high rates of depression and anxiety, particularly in the early stages of diagnosis. There is evidence to suggest that cognitive behavioural therapy (CBT) is effective for the treatment of depression amongst individuals with MS; however, there is a paucity of tailored CBT interventions designed to be offered in the newly diagnosed period. This trial is the first to assess the effectiveness and cost-effectiveness of a tailored CBT intervention compared to a supportive listening (SL) intervention amongst individuals with MS who are depressed.
\end{abstract}

Methods: ACTION-MS is a two-arm parallel group, assessor-blinded, active comparator, randomised controlled trial which will test whether a tailored CBT-based intervention compared to an SL intervention can reduce depression and related factors such as anxiety, fatigue, pain and sleep problems in those newly diagnosed with MS. Sixty participants who are within 5 years of having received a diagnosis of MS and scored within the mild to moderate range of depression on the Beck Depression Inventory (BDI-II) will be recruited from MS clinics located across three hospital sites in Melbourne, Australia. The primary outcome is depression severity using the BDI-II at post-assessment. Intervention satisfaction and acceptability will be assessed. A cost-effectiveness analysis will also be conducted. Data will be analysed on an intentionto-treat basis.

\footnotetext{
* Correspondence: litzak@unimelb.edu.au

${ }^{1}$ Melbourne School of Psychological Sciences, University of Melbourne,

Melbourne, Victoria 3010, Australia

${ }^{2}$ Department of Neurology, Royal Melbourne Hospital, Melbourne, Victoria,

Australia

Full list of author information is available at the end of the article
}

(c) The Author(s). 2020 Open Access This article is distributed under the terms of the Creative Commons Attribution 4.0 International License (http://creativecommons.org/licenses/by/4.0/), which permits unrestricted use, distribution, and reproduction in any medium, provided you give appropriate credit to the original author(s) and the source, provide a link to the Creative Commons license, and indicate if changes were made. The Creative Commons Public Domain Dedication waiver (http://creativecommons.org/publicdomain/zero/1.0/) applies to the data made available in this article, unless otherwise stated. 
(Continued from previous page)

Discussion: There is a scarcity of psychological interventions for depression targeting the newly diagnosed period. However, interventions during this time point have the potential to have a major impact on the mental and physical wellbeing of those newly diagnosed with MS. The current trial will provide data on the effectiveness of a tailored CBT intervention for the treatment of depression in those newly diagnosed with MS. Findings will also provide effect size estimates that can be used to power a later-stage multi-centre trial of treatment efficacy, and will provide information on the mechanisms underlying any treatment effects and cost-effectiveness data for delivering this intervention in outpatient MS clinics.

Trial registration: ISRCTN trials registry, ISRCTN63987586. Current controlled trials. Retrospectively registered on 20 October 2017.

Keywords: Depression, Cognitive behavioural therapy (CBT), Multiple sclerosis, Newly diagnosed, Randomised controlled trial, Anxiety

\section{Background}

High levels of depression and anxiety have been found around the time of multiple sclerosis (MS) diagnosis [1, 2] with up to $36 \%$ of individuals with MS reporting high rates of depression and anxiety in the first 2 years of diagnosis [2-4]. Point prevalence rates of depression range from 10 to $42 \%$, with up to $50 \%$ of individuals with MS experiencing depression in their lifetime $[5,6]$, which is higher than the value for any other neurological condition or chronic illness [7, 8]. Risk factors for depression amongst individuals with MS include functional status, anxiety, fatigue, cognitive impairment, pain [5, 9], losses in physical and cognitive function, occupational roles and social support [9], a reduction in previously pursued activities [10] and disease characteristics including MS illness duration, symptom severity, neurological disability and disease status [7, 11]. Demyelination and inflammation in certain parts of the brain and associated neurological changes have also been found to play a role in the development of depressive symptoms [5]. Depression in individuals with MS has been associated with poor psycho-social and treatment outcomes such as anxiety, fatigue, pain, sleep disturbances, poorer quality of life $[2,12-14]$, restricted social and economic opportunities [15], reduced medication adherence [16] and MS symptom exacerbations [17].

There is mounting evidence for the effectiveness of cognitive behavioural therapy (CBT) in treating depression amongst individuals with MS. In a recent systematic review and meta-analysis looking at interventions of depression in individuals with MS, Fiest and colleagues [18] identified eight randomised controlled trials that employed CBT-based interventions for the treatment of depression in those with MS and found that the severity of depression scores improved in all trials (standardised mean difference [SMD] - 0.45; 95\% confidence interval $[\mathrm{CI}]-0.74,-0.16)$. Despite these promising findings, research in the treatment of depression amongst individuals with MS has been limited in its methodology, with a paucity of interventions targeted towards individuals who are newly diagnosed. Early psychological intervention is important, considering rates of depression and anxiety have been found to be at clinically high levels around the time of diagnosis [1-4]. Furthermore, if depression is left untreated, it will worsen and contribute to further deterioration, having an impact on the course of MS [19], resulting in exacerbation of relapses [17], higher suicide rates [20] and lower treatment adherence [21]. Early provision of CBT may increase psychological wellbeing, lead to improvements in treatment thereby altering MS disease progression and also lead to better social and functional outcomes for individuals with MS [2].

Tailored CBT approaches adapted specifically for individuals with MS have been found to be more effective and preferred over generic CBT interventions. MS participants have found generic CBT interventions less relevant to their needs [2]. A pilot study by Kiropoulos and colleagues [2] found that a tailored early CBT intervention compared to treatment as usual was effective for treating depressive symptoms amongst patients who were within 5 years of being diagnosed with MS. Large between-group treatment effects were found for level of depression at both post-intervention $(d=1.66)$ and 20 weeks follow-up $(d=1.34)$. Those in the tailored CBT intervention also reported significantly greater improvements in quality of life, fatigue and pain management, coping, and sleep quality and reductions in anxiety with medium to large effect sizes at post-intervention and at 20 weeks follow-up $(d=1.06-0.44)$.

\section{Rationale for the present study}

Critique of previous research examining CBT for treatment of depression in those with MS includes lack of active comparator arms, small sample sizes, assessors and therapists not being blinded to participant allocation and a lack of research focused on newly diagnosed individuals $[2,22]$. We have developed a tailored CBT 
intervention for depression and other MS-related concerns including anxiety, fatigue, pain and sleep disturbance for mild and moderately depressed individuals with MS who are within 5 years of being diagnosed. The current study will be a randomised controlled trial comparing the tailored CBT intervention with supportive listening (SL). This will allow us to assess whether there are any particular benefits associated with the tailored CBT intervention compared to SL.

\section{Objectives}

The primary aim of this study is to assess the efficacy of a tailored early CBT intervention in treating depression (primary outcome) in individuals who have been newly diagnosed with MS and are experiencing mild to moderate depression. Secondary aims of the study are to examine whether those that undertake the CBT intervention will also display significant and clinically meaningful reductions in levels of anxiety. Tertiary aims of the study are to examine whether those that undertake the early tailored CBT intervention will also display significantly and clinically meaningful reductions in fatigue and pain impact, and significant improvements in sleep quality, MS-related quality of life, active coping styles, level of MS diagnosis acceptance, resilience and social support. Quarternary aims of the study are to examine the cost-effectiveness of the CBT intervention, taking into account benefits to patients, health service usage and cost of interventions.

\section{Hypotheses}

Our primary hypothesis is that there will be a significantly higher percentage of participants who achieve a clinically meaningful change of 10 points or more on the Beck Depression Inventory-II (BDI-II) [23] between baseline and post in the CBT intervention compared to those receiving the SL intervention. Our secondary hypotheses are that those in the CBT intervention will display a significantly greater change in the BDI-II total score at 20 weeks follow-up compared to those in the SL intervention; and that there will be a significantly higher percentage of participants who achieve a clinically meaningful change of 10 points or more on the State Trait Anxiety Inventory (STAI) [24] between baseline and post in the CBT intervention compared to those receiving the SL intervention. Our tertiary hypotheses are that those in the CBT intervention will display a significantly greater change in the STAI total score at 20 weeks follow-up compared to those in the SL intervention; and that those in the CBT intervention will display significant reductions in fatigue and pain impact and significant improvements in level of MS illness acceptance, MS-related quality of life, sleep quality, active coping strategies and resilience at post-intervention (8 weeks) and at 20 weeks follow-up.

\section{Methods/design}

This protocol is reported according to guidelines presented in the Consolidated Standards of Reporting Trials (CONSORT) 2010 statement for clinical trial protocols [25]. Figure 1 displays the CONSORT flow chart of participants through the trial. The Standard Protocol Items: Recommendations for Interventional Trials (SPIRIT) checklist is provided as Additional file 1.

\section{Trial design}

The ACTION-MS trial is a prospective, parallel group, assessor-blinded, active comparator, stratified, clinical trial with balanced randomisation (i.e. 1: 1).

\section{Participant recruitment}

Participants will be recruited from MS clinics located at the Royal Melbourne Hospital (RMH), St Vincent's Hospital and Western Health, Victoria, Australia. Treating neurologists, nurse co-ordinators and a researcher located at these clinics will screen patients with MS who are within 5 years of having received a diagnosis using a screening questionnaire asking two questions (Have you been feeling low most days for the last 2 weeks? and Have you lost interest or pleasure in most things?). If patients positively endorse at least one of these questions and they consent to being contacted, their contact details will be given to the trial manager for further assessment. The trial will be advertised through hospital and MS-related websites, and individuals can also self-refer to the study. The trial manager will review and confirm eligibility of participants. Individuals will be invited to an appointment for a baseline assessment at the RMH site. A total sample of 60 individuals newly diagnosed with MS will be enrolled to take part in the current study based on our pilot trial data [2].

\section{Consenting participants}

Individuals will provide informed consent to participate when they attend the baseline assessment with a clinical psychologist or registrar. No study-specific procedures will take place before written consent has been obtained. After the baseline assessment, the clinical trial manager will confirm eligibility with the participant.

\section{Inclusion and exclusion criteria}

Eligible participants will be a group of mild to moderately depressed patients (i.e. those who have scored between 14 and 28 on the BDI-II) who have been diagnosed with MS by a neurologist within the last 5 years. Exclusion criteria include (1) gross cognitive impairment that would make participation in the eight 1-h sessions of CBT distressing. Cognitive impairment will be assessed with the Brief International Cognitive Assessment for MS (BICAMS) [26]; (2) inability to speak or read English; (3) acute organic brain syndrome (e.g. 


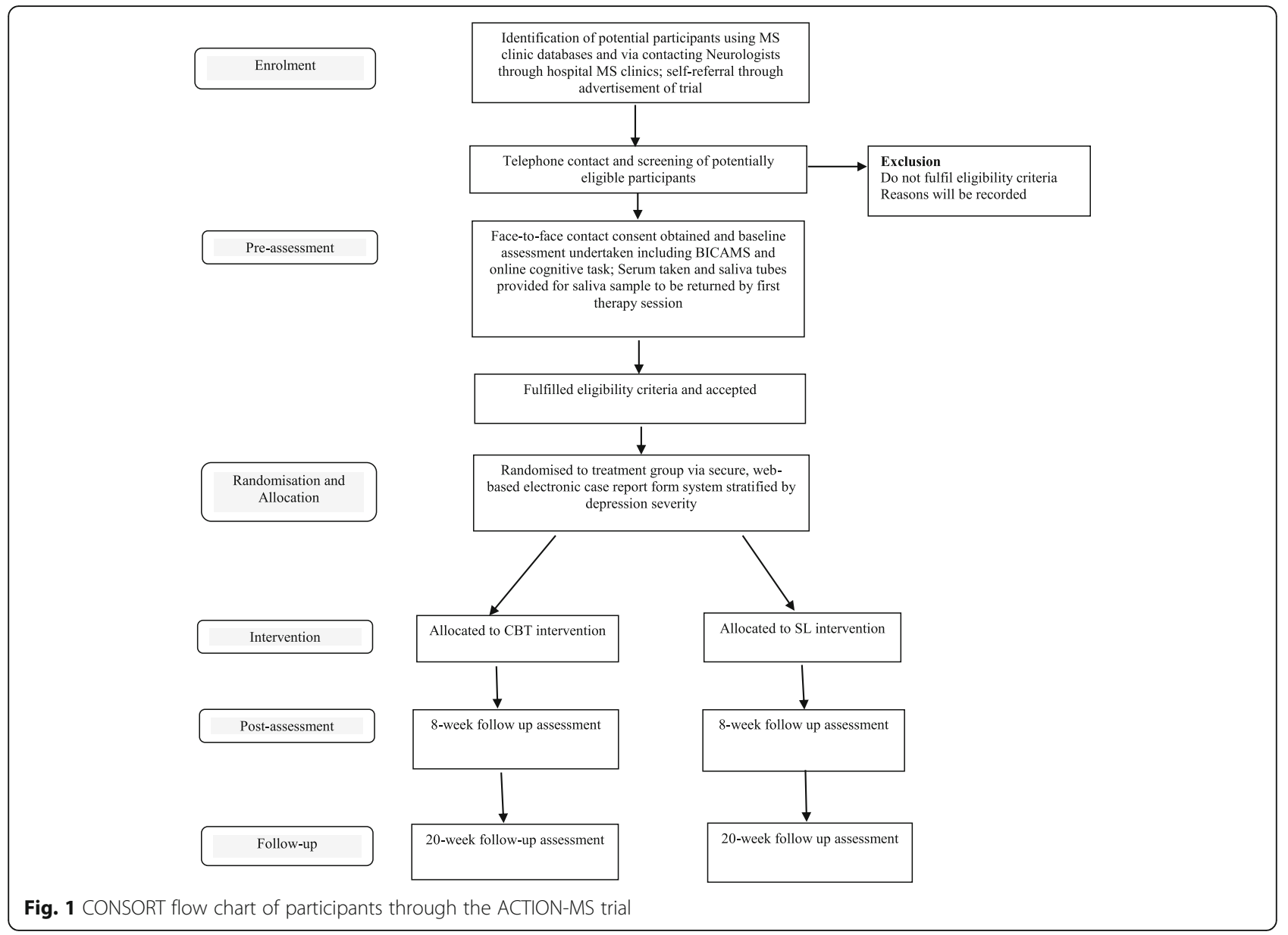

delirium); (4) serious psychological disorder (e.g. psychosis); (5) those assessed with the BDI-II (a score of 28 or more) and the Structured Clinical Interview for DSM-5 Research Version (SCID-5-RV) [27] as being severely depressed; and (6) currently undertaking psychological treatment for depression and/or anxiety. Those who are taking anti-depressant medication will be accepted after being on the medication for 2 months or longer. Any changes to disease-modifying medication will be recorded.

\section{Assessments}

Patients in both the CBT and SL groups are assessed at three time points: at baseline (T1), at the end of the intervention at 8 weeks (T2) and at 20 weeks follow-up (T3). Participants will remain part of the study for 5 months. The psychological assessment will include collection of demographic information (e.g. age, marital status, work, educational level), MS disease characteristics (e.g. diseasemodifying medications, type of MS) and psychiatric history, completion of an online study questionnaire housed on a Research Electronic Data Capture (REDCap) online system on a secure server, administration of the BICAMS [26], completion of an online cognitive task, conduction of the SCID-5-RV [27] and Serum and Saliva collection. The treating psychologists and trial manager will be unblinded to the intervention. Assessments take may take up to $2 \mathrm{~h}$ to complete.

\section{Randomisation}

Randomisation with permuted blocks of different sizes into the tailored CBT intervention and SL arms (1:1 ratio) will be undertaken via a secure, web-based Electronic Case Report Form (ECRF) system. The randomisation will be stratified by depression severity (mild and moderate depression classified using the BDI-II). Randomisation takes place after baseline assessment data has been collected. The randomisation table was created by an independent statistician and is concealed from study investigators and the assessment psychologists. Figure 1 shows the study CONSORT flow chart of participants in this trial. The trial manager will log into the ECRF system that will randomise participants into intervention group and will notify the treating psychologist about intervention allocation for the participant. 


\section{Blinding}

The clinical psychologist and registrar undertaking the baseline and outcome assessments will be blinded to participants' treatment allocation.

\section{Interventions and treatment protocol}

Participants will be randomised to either the CBT or the SL intervention. For both interventions, participants will attend weekly therapy sessions over an 8 -week period. The first session will be 90 min long; the remainder sessions will be $60 \mathrm{~min}$. All sessions will be face to face and will be undertaken in a consultation room in the RMH. Both interventions will be carried out in accordance with written and structured manuals. Participants will be issued with either a CBT or an SL client manual in their first session. The CBT and SL interventions will start within 1 week post-randomisation.

\section{Tailored cognitive behavioural therapy}

The aim of the tailored intervention is to significantly decrease levels of depression (primary outcome), anxiety (secondary outcome) and fatigue and pain impact and see improvements in levels of quality of life, fatigue and pain impact, sleep difficulties, MS illness acceptance, active coping skills, social support and resilience (tertiary outcomes). The tailored CBT intervention is derived from Beck's cognitive theoretical model for the treatment of depression in adults [28]. Table 1 provides an overview of the modules included in the tailored CBT intervention.

\section{Supportive listening}

SL includes listening skills based on the theory and counselling technique of Carl Rogers [29]. Core listening skills such as paraphrasing, reflecting, summarising and asking open-ended questions are employed. The aim is to provide the participants the opportunity to talk and express themselves in a non-judgmental, safe environment in which they experience empathy from the therapist and feel listened to. Sessions in the SL group are not structured. Participants can choose topics to discuss with their psychologist which they think are currently relevant to them. Figure 2 displays the SPIRIT schedule of enrolment, interventions and assessments.

Table 1 Overview of the eight modules included in the tailored CBT intervention

\begin{tabular}{lll}
\hline Title and description & CBT strategies \\
\hline Module 1 & Introduction to the tailored & Psycho-education on CBT model for treatment of depression and anxiety, fatigue and pain \\
& management, sleep hygiene; the link between thoughts, emotions, and behaviour; self-assessment \\
& activity identifying depressive, anxiety and MS symptoms; introduction to grief and loss model \\
& and acceptance of MS illness; self- assessment of stage of grief/loss; introduction of thought \\
& monitoring forms and provision of psycho-educational reading materials on depression, anxiety \\
& and MS and adjusting to living with MS
\end{tabular}

Module 2 and Module 3

Managing depressive and anxiety symptoms

Module 4 Challenging unhelpful thoughts

Module 5 Managing fatigue and pain

Sleep hygiene

Module 7

Problem solving

Module 8
Relapse prevention and preparing for the future
Assist client to identify link between trigger events, thoughts, emotions and behaviour; identify situations that trigger low mood and anxiety; introduction to unhelpful thinking styles and how they contribute and maintain low mood and anxiety; introduce and discuss behavioural activation strategies such as pleasant activity scheduling including physical activity; mindfulness-based controlled breathing; progressive muscle relaxation to assist in the management of negative emotions; provide guided controlled breathing, progressive muscle relaxation and sleep hygiene audio tracks; provide psycho-educational material on the benefits of controlled breathing, progressive muscle relaxation and sleep hygiene

Identifying own unhelpful thinking styles/errors using the thought monitoring form; discussion of how unhelpful thinking styles lead to negative emotions and unhelpful behaviours; introduction of how to manage and challenge unhelpful thoughts; finding alternative thoughts activity in session; provide psycho-educational material about challenging negative thoughts and coming up with alternative more helpful thoughts; provide thought monitoring, relaxation and pleasant activities forms for homework

Understanding pain and fatigue in MS from a cognitive behavioural perspective; discussion of pain and fatigue "traps"; provide psycho-educational material on fatigue and pain and depression cycle; identification of individual fatigue and pain cycle; identification of negative thoughts; identifying unhelpful thinking styles related to fatigue and pain; identification and discussion of more helpful thoughts

What is good sleep hygiene? Discussion of strategies to improve sleep such as establishing good sleep habits; provide psycho-educational material around factors that influence sleep

Introduction to problem solving; discussion of how to problem solve in session using a client's example; provide psycho-educational reading material on problem solving

Recap on all homework exercises and strategies covered in the intervention; discussion around managing depressive and anxiety symptoms, fatigue, pain and sleep problems; identifying triggers and early warning signs of depression relapse; discussion of strategies that have helped client; completion of depression relapse prevention plan in session 


\begin{tabular}{|c|c|c|c|c|c|}
\hline \multirow[b]{3}{*}{ TIMEPOINT** } & \multicolumn{5}{|c|}{ STUDY PERIOD } \\
\hline & \multirow{2}{*}{$\begin{array}{l}\text { Enrolment } \\
\text { Pre-study }\end{array}$} & \multirow{2}{*}{$\begin{array}{c}\text { Allocation } \\
\text { Visit 1 } \\
\text { Pre- } \\
\text { assessment } \\
\text { Baseline } \\
\end{array}$} & \multicolumn{2}{|c|}{ Post-allocation } & \multirow{2}{*}{$\begin{array}{c}\text { Close out } \\
\begin{array}{c}\text { Visit } 12 \\
\text { 3-month follow up } \\
\text { assessment } 20 \\
\text { weeks }\end{array} \\
\end{array}$} \\
\hline & & & $\begin{array}{c}\text { Visit } 2 \\
\text { Commencemen } \\
\text { t of treatment }\end{array}$ & $\begin{array}{c}\text { Visit } 11 \\
\text { Post-assessment } \\
8 \text { weeks }\end{array}$ & \\
\hline \multicolumn{6}{|l|}{ ENROLMENT: } \\
\hline \multirow{2}{*}{$\begin{array}{l}\text { Eligibility screen } \\
\text { Informed consent }\end{array}$} & $x$ & & & & \\
\hline & & $\mathrm{X}$ & & & \\
\hline \multirow{2}{*}{$\begin{array}{r}\text { Allocation } \\
\begin{array}{r}\text { Serum and saliva } \\
\text { collection }\end{array} \\
\end{array}$} & & $x$ & & & \\
\hline & & $x$ & & $x$ & $x$ \\
\hline \multicolumn{6}{|l|}{ INTERVENTIONS: } \\
\hline \multicolumn{6}{|l|}{$C B T$} \\
\hline \multicolumn{6}{|l|}{$S L$} \\
\hline \multicolumn{6}{|l|}{ ASSESSMENTS: } \\
\hline \multirow{3}{*}{$\begin{array}{r}\text { SCID-5-RV } \\
\text { BICAMS } \\
\text { Online Cognitive } \\
\text { Task }\end{array}$} & & $x$ & & $x$ & $x$ \\
\hline & & $\mathrm{X}$ & & $x$ & $x$ \\
\hline & & & & & \\
\hline BDI-II & & $x$ & & $x$ & $x$ \\
\hline \multirow{2}{*}{ STAI } & & $x$ & & $x$ & $\mathrm{X}$ \\
\hline & & $\mathrm{X}$ & & $x$ & $x$ \\
\hline MFIS-5 & & $x$ & & $x$ & $x$ \\
\hline PES & & $\mathrm{X}$ & & $x$ & $\mathrm{X}$ \\
\hline$M S-Q O L$ & & $x$ & & $x$ & $\mathrm{X}$ \\
\hline WOC & & $x$ & & $x$ & $x$ \\
\hline ACHCS & & $x$ & & $x$ & $x$ \\
\hline$R S A$ & & $\mathrm{X}$ & & $x$ & $x$ \\
\hline$T A Q$ & & & & & \\
\hline$T S Q$ & & & & $x$ & \\
\hline
\end{tabular}

Fig. 2 SPIRIT schedule of enrolment, interventions and assessments. CBT cognitive behavioural therapy, SL supportive listening, SCID-5RV Structured Clinical Interview for DSM-5 Research Version, BICAMS Brief International Cognitive Assessment for MS, BDI-II Beck Depression Inventory-II, STAI State Trait Anxiety Inventory, MFIS-5 Modified Fatigue Impact Scale-5 item version, PES Pain Effects Scale, MS-QOL multiple sclerosis-related quality of life measure, WOC 66 -item Ways of Coping questionnaire, ACHCS Acceptance of Chronic Health Conditions Scale, RSA Resilience Scale for Adults, PSSS Perceived Stress Scale, TAQ Therapeutic Alliance Questionnaire, TSQ Therapy Satisfaction Questionnaire

\section{Training and supervision of therapists providing the interventions}

Therapists will be registered clinical psychologists and registrars with training in CBT. All therapists will complete a 2-day workshop during which they will receive training in the two therapy techniques, with a day devoted to each of the CBT and SL interventions. All therapists will follow strict manualised protocols under the supervision of the trial manager.

\section{Standardisation of the intervention}

Standardisation of the intervention will be achieved by (1) use of a detailed intervention protocol which includes a therapist and client manual; (2) external 
monitoring of protocol compliance with routine feedback, through weekly supervision by the trial manager with the treating psychologists; (3) a protocol reminder system on our web-based data entry and management tool; and (4) a Data Safety and Monitoring Committee (DSMC) who will review protocol compliance. The DSMC will comprise an independent psychologist, neurologist and statistician who will be in contact with the principal researcher and trial manager.

\section{Treatment fidelity}

All intervention sessions will be recorded and used in supervision to provide feedback to therapists and to ensure treatment fidelity. We aim to take a $25 \%$ sample of intervention sessions for those in the CBT and SL groups from participants who agree to be recorded based on a random sample generated from an algorithm that takes into account session number, therapist and patient gender. Quality of CBT and SL administered will be assessed using the Cognitive Therapy Rating Scale [30] and a Supportive Listening Rating Scale. In addition, therapists in each condition will be asked to keep a selfreported fidelity checklist of all sessions.

\section{Outcome measures}

\section{Primary outcome measure}

The primary outcome is the percentage of participants achieving the clinically meaningful change of 10 points or more on the BDI-II between baseline and postintervention in the CBT intervention. Level of depression will be measured with the BDI-II.

\section{Secondary outcome measures}

Secondary outcomes are the magnitude of change between baseline and 20 weeks on the BDI-II in the CBT intervention with the level of depression measured with the BDI-II and the magnitude of change between baseline and post-intervention on the STAI. Level of anxiety will be measured with the STAI.

\section{Tertiary outcome measures}

Tertiary outcomes are the magnitude of change between baseline and post-intervention on fatigue and pain impact, MS-related quality of life, sleep quality, MS diagnosis acceptance, active coping styles, resilience and perceived social support in the early CBT intervention. Fatigue impact will be measured with the Modified Fatigue Impact Scale-5 item version (MFIS-5) [31]. Pain impact will be measured using the 6-item Pain Effects Scale [31]. Sleep quality will be measured with the Pittsburgh Sleep Quality Index [32]. MS-related quality of life is measured using the 54-item MS-related quality of life measure [33]. Coping styles will be examined using the 66-item Ways of Coping questionnaire [34]. Acceptance of MS illness will be measured using the 10-item Acceptance of Chronic Health Conditions Scale [35]. Level of resilience will be measured with the 33-item Resilience Scale for Adults [36], and Perceived Social Support will be measured using the 12-item Scale of Perceived Social Support [37].

\section{Process measures}

The Therapeutic Alliance Questionnaire [38] will be given to participants at each session, and they will be asked to complete and place it in an envelope which will be given to the therapist the following week. At postintervention we will ask participants to complete a Therapy Satisfaction Questionnaire to provide information about the acceptability of the CBT intervention.

\section{Saliva and serum samples}

Participants will be asked to provide serum samples at baseline, post-intervention (8 weeks) and at 20 weeks follow-up. Additional consent for their use in future related research will be obtained.

\section{BICAMS}

We will collect test-retest reliability data for BICAMS from all participants enrolled in the trial. Participants will complete the BICAMS at the baseline assessment and again 1 week after this and prior to commencing their first therapy session. Additional consent for this data to be used in future related research will be obtained.

\section{Sample size calculation}

The current study is powered for the primary outcome of the proportion of patients who achieve a clinically significant change of 10 points or more on the BDI-II between baseline and post-treatment in the CBT intervention compared to those receiving the SL intervention. Based on pilot data by Kiropoulos and colleagues [2], the current study will aim to recruit 30 participants in each treatment arm (CBT and SL). The pilot results also revealed that $33 \%$ of newly diagnosed individuals screened did not meet eligibility to participate in the trial. Therefore, the current study will aim to screen 90 patients newly diagnosed with MS across three MS clinics in three hospital sites and via advertisements in order to obtain a total sample size of 60 . This number will yield $90 \%$ power using an $\alpha=0.05$ threshold to observe a conservatively estimated $45 \%$ difference in proportion of patients achieving a favourable primary outcome. The sample size has also been inflated for drop-outs throughout the 20-week follow-up study. 


\section{Planned statistical analysis Effectiveness analysis}

The primary outcome analysis will be an intention-totreat between-group comparison of the proportion of patients who achieve a clinical and meaningful change of 10 points or more on the BDI-II between baseline and post-intervention adjusted for baseline depression severity using a logistic regression model. Baseline characteristics will be presented by randomised group without formal statistical tests. Secondary and tertiary outcome analyses will be undertaken using appropriate regression models adjusted for baseline depression severity. Longitudinal changes will be examined using regression models. No formal interim analyses for either efficacy or safety are planned, but safety outcomes will be continuously monitored.

\section{Health economics evaluation}

Participants will be asked to record service use including all types and duration of hospital admissions, frequency of outpatient hospital appointments, MS-related community service use, anti-depressant and anti-anxiety medication use and cost and paid and unpaid work using the Client Service Receipt Inventory [39]. Healthrelated quality of life data and quality-adjusted life years (QALYs) will be assessed using the health-related quality of life measure (EuroQoL-5D) [40].

\section{Trial monitoring and management and patient safety}

The study will be co-ordinated by a trial manager who will be a clinical psychologist. Trial monitoring and management will be undertaken by (1) a steering committee who will meet once a year to discuss the overall conduct of the trial and will include the lead investigator and key investigators from the recruitment sites, the trial manager and the statistician; (2) a management committee who will meet fortnightly to monthly to discuss recruitment issues and will include the trial manager and the key investigators from the hospital sites; and (3) safety monitoring, as performed by the independent DSMC who will provide advice regarding the safety of the trial to the steering committee and will consist of a clinician, a psychologist and an independent statistician. We will continuously monitor serious adverse events. All adverse events will be prospectively classified as serious or not serious. An independent clinical adjudication committee adjudicates all serious adverse events for this trial for subsequent reporting to the DSMC. In the unlikely case of an adverse event, this will be documented by the principal researcher and trial manager. Precautions have been taken to reduce the likelihood of adverse events occurring such as the exclusion of patients who are acutely suicidal or severely depressed. The interventions are delivered by clinical psychologists or registrars under the weekly clinical supervision of the trial manager. In the case of any adverse events as a result of taking part in either of the interventions, participation in the trial will be discontinued. Serious adverse events will be reported to the ethics committee. Any adverse events will be reported in the trial paper.

\section{End of study}

The end of the study is defined as the last data collection during the last 20 weeks follow-up visit from the last participant after the CBT or SL intervention, whichever comes last.

\section{Data management, protection, storage and archiving of study documents}

Access to participant data will be restricted to the principal researcher and appropriate study staff as needed. All laboratory specimens and questionnaire forms will be identified using unique participant ID numbers to maintain participant confidentiality. All records including the serum and saliva samples will be kept in secure storage areas with limited access to study staff only. Personal data and anonymised data files will be stored in locked filing cabinets for hard copies and in a secure online database housed on the University of Melbourne secured server with access via password-protected computers by study staff only. All study documentation will be kept for a minimum of 5 years from the protocoldefined end-of-study point in the University of Melbourne archive. All documentation will be destroyed after this date.

\section{Participant adherence}

Adherence to the CBT intervention will be defined as attendance of the baseline assessment session and attendance and completion of at least four CBT sessions with a psychologist. We will also monitor patient adherence to the CBT intervention through completion of a patient satisfaction questionnaire at the 8 weeks post-assessment. Participant drop-out and reason will be recorded.

\section{Withdrawal procedures}

Participants will have the option to withdraw at any time during the study period of 5 months. The participant will need to request this formally, and the reason for withdrawing from the study will be recorded by the trial manager. Participant data collected prior to the withdrawal will be used with the permission of the participant. A participant can be withdrawn by the chief investigator should the need arise throughout the study period, and this will also be recorded. 


\section{Patient and public involvement}

Patients have been involved in the development of the research question and the prioritisation of depression and related factors in the newly diagnosed period as a subject for research. In our pilot trial, patients were involved in a semi-structured qualitative interview where they gave feedback about the content of the tailored CBT intervention and their experience of participating in the trial using the tailored CBT intervention manual [2]. Patients provided feedback about the feasibility, usefulness, appropriateness and content of the tailored CBT intervention [2]. We will send all participants a report describing the findings and their implications.

\section{Discussion}

Depression is common in individuals newly diagnosed with MS $[1,2]$ and, if left untreated, it will contribute to further deterioration, having an impact on the course of MS [19] and immune functioning [5], result in the exacerbation of MS relapses [17], contribute to higher suicide rates [20] and possibly affect adherence to medical advice and treatments $[16,21]$. There is a scarcity of psychological interventions for depression targeting the newly diagnosed period. Such interventions have the potential to have a major impact on the mental and physical wellbeing of those newly diagnosed with MS [2]. Our pilot trial data has shown the tailored CBT intervention to significantly reduce depressive and anxiety symptoms and provide an increase in quality of life and better management of fatigue, pain and sleep disturbances [2] in those newly diagnosed with MS. The current trial will test the efficacy of a tailored CBT intervention for depression. It will provide a range of effect size estimates that can be used to power a later-stage multi-centre trial of treatment efficacy, it will inform the mechanisms underlying any treatment effects and will provide acceptability and cost-effectiveness data for delivering this intervention through outpatient MS clinics.

\section{Trial status}

The ACTION-MS trial began recruitment on 14 June 2017 using protocol version number 1, 14 June 2016. It is anticipated that recruitment will be ongoing until December 2021. It is expected that data collection will be completed by April 2022.

\section{Supplementary information}

Supplementary information accompanies this paper at https://doi.org/10. 1186/s13063-019-4018-8.

Additional file 1. SPIRIT 2013 checklist: recommended items to address in a clinical trial protocol and related documents.

\section{Abbreviations}

BDI-II: Beck Depression Inventory-II; BICAMS: Brief International Cognitive Assessment for MS; CBT: Cognitive behavioural therapy;
CONSORT: Consolidated Standards of Reporting Trials; DSMC: Data Safety and Monitoring Committee; ECRF: Electronic Case Report Form; MS: Multiple sclerosis; QALY: Quality adjusted life year; RMH: Royal Melbourne Hospital; SCID-5-RV: Structured Clinical Interview for DSM-5 Research Version;

SL: Supportive listening; SPIRIT: Standard Protocol Items: Recommendations for Interventional Trials; STAI: State Trait Anxiety Inventory

\section{Acknowledgements}

The authors thank the Clinical Trials Centre at the Royal Melbourne Hospital, Australia, who provided in-kind support to run this trial, and the MS clinics at the Royal Melbourne Hospital, St Vincent's Hospital and Western Health, who assisted in recruitment. We would like to thank all the research psychologists involved in the assessment of all participants and the delivery of the interventions to participants and all treating neurologists at the MS clinics located at the various hospital sites. We would also like to thank MS Research Australia and MS Society Australia for advertising the clinical trial.

\section{Authors' contributions}

LK conceptualised and initiated this study. LK, TKi and LC contributed to the design of the study. LK developed the assessment and intervention content. JT further developed the intervention content. LK is responsible for randomisation and allocation of participants via REDCap. AVDW suggested the inclusion of the online cognition task. LK wrote the draft of the manuscript. LK, TKi, TKa, LT, EM, TW and AVDW have contributed to the recruitment of participants. All authors approved the final version of the manuscript. NSO is responsible for the analysis of the serum and saliva samples.

\section{Funding}

This work was supported by a Multiple Sclerosis Research Australia Project Grant (Grant Number 15-013). This funding source has no influence on the design of the study and has not influenced its execution, analyses, interpretation of the data or decision to submit results.

\section{Availability of data and materials}

The datasets used and/or analysed during the current study are available from the corresponding author on reasonable request.

\section{Ethics approval and consent to participate}

The study protocol was approved by the Melbourne Health Human Research Ethics Committee at its meeting on 7 August 2016, and research governance approval was received on 17 May 2017. Approval was also obtained from St Vincent's Hospital on 11 April 2017 and from Western Health on 26 April 2019 for recruitment at these sites. Dissemination of findings will be done via publications and presentation at scientific meetings and conferences. Written informed consent will be obtained from all participants including consent for the recording of intervention sessions for quality assurance and treatment fidelity purposes and for serum and saliva collection.

\section{Consent for publication}

Not applicable.

Competing interests

The authors declare that they have no competing interests.

\section{Author details}

${ }^{1}$ Melbourne School of Psychological Sciences, University of Melbourne, Melbourne, Victoria 3010, Australia. ${ }^{2}$ Department of Neurology, Royal Melbourne Hospital, Melbourne, Victoria, Australia. ${ }^{3}$ Florey Institute of Neuroscience and Mental Health, Melbourne, Victoria, Australia. ${ }^{4}$ Rehabilitation and Neuroimmunology, Department of Neuroscience, St Vincent's Hospital, Melbourne, Victoria, Australia. ${ }^{5}$ Department of Neurology, Western Health, Sunshine, Victoria, Australia. ${ }^{6}$ Melbourne Dental School, University of Melbourne, Carlton, Victoria, Australia. ${ }^{7}$ Department of Neurosciences, Monash University, Melbourne, Victoria, Australia. 
Received: 20 October 2019 Accepted: 19 December 2019

Published online: 20 January 2020

\section{References}

1. Giordano A, Granella F, Lugaresi A, Martinelli V, Trojano M, Confalonieri P, Radice D, Solari A. Anxiety and depression in multiple sclerosis patients around diagnosis. J Neurol Sci. 2011;307:86-91.

2. Kiropoulos LA, Kilpatrick T, Holmes A, Threader J. A pilot randomized controlled trial of a tailored cognitive behavioural therapy based intervention for depressive symptoms in those newly diagnosed with multiple sclerosis. BMC Psychiatry. 2016;16:435.

3. Janssens A, van Dorn P, de Boer J, van der Meche F, Passchier J, Hintzen R. Impact of recently diagnosed multiple sclerosis on quality of life, anxiety, depression and distress of patients and partners. Acta Neurol Scand. 2003; 108:389-95.

4. Tan-Kristanto S, Kiropoulos LA. Resilience, self-efficacy, coping styles and depressive and anxiety symptoms in those newly diagnosed with multiple sclerosis. Psychol Health Med. 2015;14:1-11.

5. Arnett PA, Barwick FH, Beeney JE. Depression in multiple sclerosis: review and theoretical proposal. J Int Neuropsychol Soc. 2008;14:691-724.

6. Siegert RJ, Abernathy DA. Depression in multiple sclerosis. J Neurol Neurosurg Psychiatry. 2005;76:469-75.

7. Chwastiak L, Ehde DM, Gibbons LE, Sullivan M, Bowen JD, Kraft GH. Depressive symptoms and severity of illness in multiple sclerosis: epidemiologic study of a large community sample. Am J Psychiatry. 2002; 159:1862-8

8. Suh Y, Motl RW, Mohr DC. Physical activity, disability and mood in the early stages of multiple sclerosis. Disabil Health J. 2010;3:93-8.

9. Gay MC, Vrignaud P, Garitte C, Meunier C. Predictors of depression in multiple sclerosis patients. Acta Neurol Scand. 2010;121:161-70.

10. Mohr DC, Cox D. Multiple sclerosis: empirical literature for the clinical psychologist. J Clin Psychol. 2001;57:479-99.

11. Mclvor GP, Riklan M, Reznikoff M. Depression in multiple sclerosis as a function of length and severity of illness, age, remissions, and perceived social support. J Clin Psychol. 1984;40:1028-33.

12. D'Alisa S, Miscio G, Baudo S, Simone A, Tesio L, Mauro A. Depression in the main determinant of quality of life in multiple sclerosis: a classificationregression (CART) study. Disabil Rehabil. 2006;28:307-14.

13. Mitchell AJ, Benito-Leon J, Gonzalez JM, Rivera-Navarro J. Quality of life and its assessment in multiple sclerosis: integrating physical and psychological components of wellbeing. Lancet Neurol. 2005;4:556-66.

14. Wang JL, Reimer MA, Metz LM, Patten SB. Major depression and quality of life in individuals with multiple sclerosis. Int J Psychiatry Med. 2000; 30:309-17.

15. Green G, Todd J. 'Restricting choices and limiting independence': social and economic impact of multiple sclerosis upon households by level of disability. Chronic IIIn. 2008:4:160-72.

16. Pozzilli, Schweikert, Ecari, Oentrich. BetaPlus Study group. Supportive strategies to improve adherence to IFN $\beta-1 b$ in multiple sclerosis-results of the BPlus observational cohort study. J Neurol Sci. 2011;307:120-6.

17. Lu CZ, Jensen MA, Arnason BG. Interferon gamma- and interleukin-4secreting cells in multiple sclerosis. J Neuroimmunol. 1993;46:123-8.

18. Fiest KM, Walker JR, Bernstein CN, et al. Systematic review and meta-analysis of interventions for depression and anxiety in persons with multiple sclerosis. Mult Scler Relat Disord. 2016;18:96-104.

19. Mohr DC, Goodkin DE. Treatment of depression in multiple sclerosis, review and meta-analysis. Clin Psychol. 1999;6:1-9.

20. Sadovnick AD, Eisen K, Ebers GC, Paty DW. Cause of death in patients attending multiple sclerosis clinics. Neurology. 1991;41:1193-6.

21. Mohr DC, Goodkin DE, Likosky W, Gatto N, Neilley LK, Griffen C, Stiebling B. Therapeutic expectations of patients with multiple sclerosis upon initiating interferon beta-1b: relationship to adherence to treatment. Mult Scler. 1996; 2:222-6.

22. Hind P, Cotter J, Thake A, Bradburn M, Cooper C, Isaac C, House A. Cognitive behavioural therapy for the treatment of depression in people with multiple sclerosis: a systematic review and meta-analysis. BMC Psychiatry. 2014;14:5.

23. Beck AT, Steer RA, Brown GK. Manual for the Beck Depression Inventory-II. San Antonio: The Psychological Corporation; 1996.

24. Spielberger CD, Gorsuch RL, Lushene RE. Manual for the State-Trait Anxiety Inventory. Palo Alto: Consulting Psychologists Press; 1970.
25. Schulz KF, Altman DG, Moher D, for the CONSORT Group. CONSORT 2010 Statement: updated guidelines for reporting parallel group randomised trials. BMJ. 2010;340:C332.

26. Benedict RHB, Amato MP, Boringa J, et al. Brief International Cognitive Assessment for MS (BICAMS): international standards for validation. BMC Neurology. 2012;12:55.

27. First MB, Williams JBW, Karg RS, Spitzer RL. Structured clinical interview for DSM-5-Research Version (SCID-5 for DSM-5, Research Version; SCID-5-RV). Arlington: American Psychiatric Association; 2015.

28. Beck AT, Rush AJ, Shaw BE, Emery G. Cognitive therapy of depression. New York: Guilford Press; 1979.

29. Rogers C. Client-centred therapy: its current practice, implications and theory. London: Constable; 1951.

30. Young JE, Beck AT. Cognitive Therapy Scale rating manual. Bala Cynwyd: Beck Institute for Cognitive Behavior Therapy; 1980.

31. Ritvo PG, Fischer JS, Miller DM, Andrews H, Paty DW, LaRocca NG. Multiple Sclerosis Quality of Life Inventory: a user's manual. NY: National Multiple Sclerosis Society; 1997. https://www.nationalmssociety.org/ NationalMSSociety/media/MSNationalFiles/Brochures/MSQLI_-A-User-sManual.pdf. Accessed 17 Dec 2019.

32. Buysse DJ, Reynolds CF 3rd, Monk TH, Berman SR, Kupfer DJ. The Pittsburgh Sleep Quality Index: a new instrument for psychiatric practice and research. Psychiatry Res. 1989;28:193-213.

33. Vickrey BG, Hays RD, Harooni R, Myers LW, Ellison GW. A health-related quality of life measure for multiple sclerosis. Qual Life Res. 1995;4:187-206.

34. Folkman S, Lazarus RS. Manual for the Ways of Coping Scale. Palo Alto: Consulting Psychologists Press; 1988.

35. Stuifbergen A, Becker H, Blozis S, et al. Conceptualization and development of the Acceptance of Chronic Health Conditions scale. Issues Ment Health Nurs. 2008;29:101-14.

36. Hjemdal O, Friborg O, Braun S, Kempenaers C, Linkowski P, Fossion P. Resilience Scale for Adults: construct validity and measurement in a Belgian sample. Int J Test. 2011;11:53-70.

37. Zimet GD, Dahlem NW, Zimet SG, Farley GK. The Multidimensional Scale of Perceived Social Support. J Pers Assess. 1988;52:30-41.

38. Luborksy L, Barber JP, Siqueland L, Johnson S, Najavits LM, Frank A, Daley D. The revised Helping Alliance Questionnaire (HAq-II): psychometric properties. J Psychother Pract Res. 1996;5:260-71.

39. Curtis L, Netten A. Unit Costs of Health and Social Care 2006. Canterbury: Personal Social Services Research Unit; 2006. https://www.pssru.ac.uk/ project-pages/unit-costs/unit-costs-2006/. Accessed 17 Dec 2019

40. Beecham J, Knapp M. Costing psychiatric interventions. In: Thornicroft, editor. Measuring mental health needs. $2^{\text {nd }}$ ed. London: Royal College of Psychiatrists; 2001. http://citeseerx.ist.psu.edu/viewdoc/download?doi= 10.1.1.618.1930\&rep=rep1\&type=pdf. Accessed 17 Dec 2019.

\section{Publisher's Note}

Springer Nature remains neutral with regard to jurisdictional claims in published maps and institutional affiliations.

\section{Ready to submit your research? Choose BMC and benefit from:}

- fast, convenient online submission

- thorough peer review by experienced researchers in your field

- rapid publication on acceptance

- support for research data, including large and complex data types

- gold Open Access which fosters wider collaboration and increased citations

- maximum visibility for your research: over $100 \mathrm{M}$ website views per year

At BMC, research is always in progress.

Learn more biomedcentral.com/submissions 